\title{
Complete section of the left vagus nerve does not preclude the efficacy of vagus nerve stimulation: illustrative case
}

\author{
Alice Noris, MD, ${ }^{1}$ Paolo Roncon, PhD, ${ }^{2}$ Simone Peraio, MD, ${ }^{1}$ Anna Zicca, MD, ${ }^{3}$ Matteo Lenge, PhD, ${ }^{1}$ Andrea Di Rita, MD, ${ }^{1}$ \\ Lorenzo Genitori, MD, ${ }^{1}$ and Flavio Giordano, MD ${ }^{1}$
}

${ }^{1}$ Neurosurgery Unit, Department of Neurosciences, and ${ }^{3}$ Anesthesiology and Intensive Care Unit, Meyer Children's Hospital, Florence, Italy; and ${ }^{2}$ Medical Affairs Department, Sorin Group Italia, LivaNova PLC-Owned Subsidiary, Milan, Italy

\begin{abstract}
BACKGROUND Vagus nerve stimulation (VNS) represents a valid therapeutic option for patients with medically intractable seizures who are not candidates for epilepsy surgery. Even when complete section of the nerve occurs, stimulation applied cranially to the involved nerve segment does not preclude the efficacy of VNS. Complete vagus nerve section with neuroma causing definitive left vocal cord palsy has never been previously reported in the literature.
\end{abstract}

OBSERVATIONS Eight years after VNS implant, the patient experienced worsening of seizures; the interrogation of the generator revealed high impedance requiring surgical revision. On surgical exploration, complete left vagus nerve section and a neuroma were found. Vocal cord atrophy was found at immediate postoperative laryngeal inspection as a confirmation of a longstanding lesion. Both of these events might have been caused by direct nerve injury during VNS surgery, and they presented in a delayed fashion.

LESSONS VNS surgery may be complicated by direct damage to the left vagus nerve, resulting in permanent neurological deficits. A complete section of the nerve also enables an efficacious stimulation if applied cranially to the involved segment. Laryngeal examination should be routinely performed before each VNS surgery to rule out preexisting vocal cord dysfunction.

https://thejns.org/doi/abs/10.3171/CASE21128

KEYWORDS vagus nerve stimulation; refractory epilepsy; neuroma; vocal cord palsy

Vagus nerve stimulation (VNS) was approved as adjunctive therapy to treat drug-resistant epilepsy (DRE) in Europe in 1994 and in the United States in 1997. ${ }^{1,2}$ Its efficacy has also been investigated for other conditions, and it is approved for the treatment of difficult-to-treat depression. ${ }^{3}$ VNS consists of electrical stimulation of the left vagus nerve by means of an electrode connected to an implanted device that generates impulses (VNS physicians manual). ${ }^{4}$ A study on adult patients who received DRE implantation with VNS reported that $64 \%$ of individuals had a seizure reduction of $\geq 50 \%$ after 59 months of observation. ${ }^{2}$ Similar results have also been reported in children, with $65 \%$ of patients experiencing a $\geq 50 \%$ seizure reduction at 64 months' follow-up. ${ }^{2}$ Although the therapeutic effect of VNS therapy is proven clinically, the mechanisms of action of VNS are not fully described, and multiple mechanisms that result in inhibiting cortical electrical activity seem to be involved. ${ }^{5}$ Side effects related to VNS are usually mild, intermittent, perioperative, and well-tolerated. The main ones are voice alterations, cough, throat discomfort, dyspnea, sleep apnea, vascular injury, and cardiac arrhythmias. ${ }^{4-7}$ Hoarseness is the most common adverse event, occurring in up to $37 \%$ of patients, and it is generally well-tolerated. ${ }^{5-8}$ Vocal cord paralysis has been reported rarely and only in relation to stimulator malfunction or as a surgical complication. ${ }^{5}$ Here, we describe the first case of a patient with a complete vagus nerve section and proximal-end neuroma 8 years after VNS implantation, causing a unilateral complete left vocal cord paralysis.

\section{Illustrative Case}

History and Presentation

A 27-year-old woman presented with medically intractable epilepsy. Her previous medical history was uneventful, except for a slight psychomotor

ABBREVIATIONS AED = antiepileptic drug; DRE = drug-resistant epilepsy; IPG = implantable pulse generator; VNS = vagus nerve stimulation. INCLUDE WHEN CITING Published July 19, 2021; DOI: 10.3171/CASE21128.

SUBMITTED February 25, 2021. ACCEPTED April 2, 2021.

(C) 2021 The authors, CC BY-NC-ND 4.0 (http://creativecommons.org/licenses/by-nc-nd/4.0/). 
delay. Seizures began at 10 years of age and progressively worsened: they were characterized by drop attacks and partial complex seizures with loss of consciousness, speech arrest, hypersalivation, and right arm weakness; she experienced two to three episodes per week. She was initially prescribed phenytoin $(50 \mathrm{mg}$ in the morning and $100 \mathrm{mg}$ in the evening), clobazam $10 \mathrm{mg}$ three times a day and valproic acid (first $100 \mathrm{mg}$ twice a day and then $200 \mathrm{mg}$ twice a day). The latter was subsequently replaced with lacosamide $(200 \mathrm{mg}$ in the morning, $50 \mathrm{mg}$ at noon, and $200 \mathrm{mg}$ in the evening) due to inefficacy and side effects of valproic acid. Video electroencephalogram showed multiple bilateral spikes and diffuse spike-waves, especially in temporal regions. Magnetic resonance imaging investigations showed only bilateral parietooccipital polymicrogyria; genetic workup was negative. Antiepileptic drug (AED) dosages were progressively increased due to poor clinical response.

In 2005, at 20 years of age, she underwent a first implantation of the VNS model E102 Neuro-Cybernetic Prosthesis system (LivaNova PLC) in another hospital; neither intra- nor postoperative complications were reported. However, she experienced hoarseness and dysphonia immediately after surgery and before stimulation of the vagus nerve. Despite a $30 \%$ reduction of seizures, she continued to suffer from drop attacks due to atonic/tonic seizures. After a multidisciplinary evaluation at our Epilepsy Surgery Center, in 2012, she underwent anterior corpus callosotomy, leading to a complete resolution of drop attacks. One year later, the VNS testing showed high impedance and revealed that the implantable pulse generator (IPG) had almost reached the end of its life. The patient reported no history of trauma but a relapse of drop attacks and persistent partial motor seizures in the right arm. She was still complaining of hoarseness. On the basis of these findings, surgical revision of the VNS device was offered to the patient. At that time, the patient was receiving four AEDs (carbamazepine $400 \mathrm{mg}$ three times a day and clobazam $10 \mathrm{mg}$, phenytoin $50 \mathrm{mg}$, and lacosamide $200 \mathrm{mg}$ twice a day).

\section{Operative Management}

The previous incision was reopened on the left laterocervical region. The electrode coils and leads were wrapped around the vagus nerve, along with dense and tough scar tissue. Surgery was performed under microscopic view with ultrasharp low-voltage monopolar and blunt dissection. A complete section of the left vagus nerve was discovered distally to the electrodes; the proximal end of the cut nerve presented a cauliflower-like neuroma, while the distal end was hidden by scar tissue and probably retracted (Fig. 1). The electrodes were carefully dissected out of the nerve in a piecemeal fashion to avoid excessive pulling and/or manipulation of the nerve. Hypertrophy of the vagus nerve from scar formation and indentation at the site of the electrode coils were present. New electrodes were positioned around a more cranial-naïve segment, and the vagus nerve was restored to its anatomical position in the carotid sheath. Finally, the IPG was replaced in the same left infraclavicular subcutaneous pouch with a new VNS device model E103 Neuro-Cybernetic Prosthesis system (LivaNova PLC). A regular impedance test (1300 ohm) was carried out.

\section{Postoperative Course}

The postoperative period was uneventful except for the preexisting hoarseness. A flexible laryngoscopy was performed the first day after surgery and showed a complete paralysis of the left vocal cord; its atrophy proved that the damage must have been very old and not a result of direct injury during revision surgery. The patient

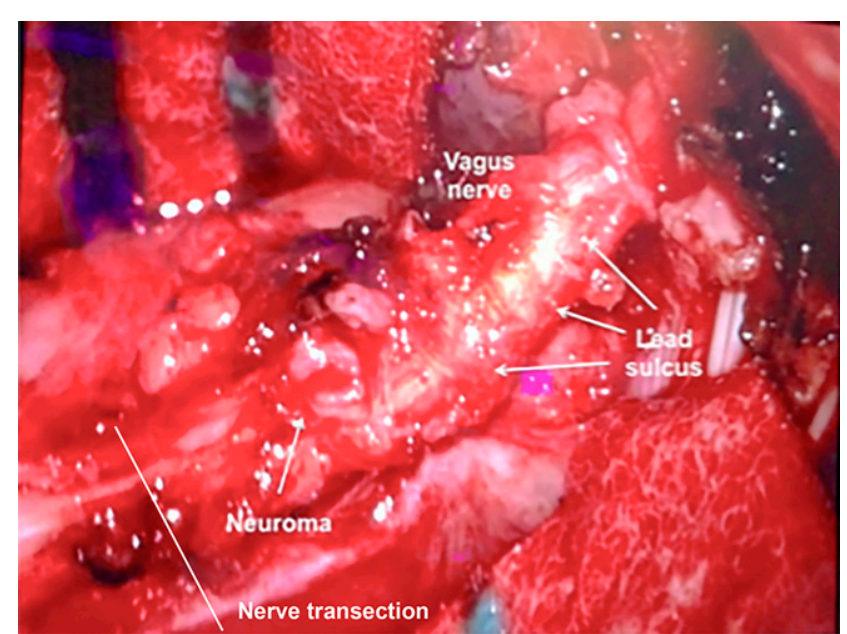

FIG. 1. Intraoperative photograph showing the left vagus nerve thickened by scar tissue and completely sectioned. The indentation of the electrodes, the neuroma in the proximal end of the cut nerve, and the nerve transection are visible.

subsequently underwent medialization laryngoplasty, improving her phonation. The VNS was activated 1 month later with gradual titration to standard parameters $(2.50 \mathrm{mV} ; 30 \mathrm{sec}$ ON, $5 \mathrm{~min}$ OFF; 500 $\mathrm{msec} ; 30 \mathrm{~Hz}$ ); the impedance was regular. The patient experienced about $70 \%$ frequency reduction of seizures. She was able to gradually reduce AED number and dosages. In 2016, she underwent a second VNS system revision for high impedance due to new scar formation; both the electrodes and the IPG were replaced with a model E104 Neuro-Cybernetic Prosthesis system (Cyberonics). A regular impedance test (1540 ohm) was carried out. Seizure frequency reduced further, and at last follow-up, 5 years later, epilepsy is still currently well-controlled by the VNS therapy and two AEDs (carbamazepine $400 \mathrm{mg}$ three times a day and lacosamide $200 \mathrm{mg}$ in the morning, $100 \mathrm{mg}$ at noon, and $100 \mathrm{mg}$ in the evening). She uses clobazam and clonazepam as rescue medication as needed.

\section{Discussion \\ Observations}

VNS therapy is an effective treatment for medically intractable epilepsy, resulting in a $\geq 50 \%$ seizure reduction in about $65 \%$ of adult and pediatric populations. ${ }^{2}$ It has been considered safe because side effects are usually mild, temporary, and well-tolerated. ${ }^{6}$ We described a case of a complete left vagus nerve section with proximal-end neuroma found during surgery for complete VNS revision. After the electrodes were carefully dissected from the nerve and the entire system was replaced with coils lodged in a more cranial position, the therapeutic effect of VNS was restored. To our knowledge, this is the first case demonstrating a complete anatomical interruption of the nerve that does not impede the efficacy of VNS stimulator placed upwards. Moreover, based on our information, only one case has been reported in the literature describing a vagus nerve injury with partial section and neuroma formation occurring after mild blunt neck trauma. ${ }^{9}$

\section{General Complications}

Complications in VNS surgery can be distinguished in early and delayed complications. ${ }^{5-7}$ The first ones include intraoperative bradycardia, 
which mainly occur during lead impedance testing and account for $0.1 \%$ of cases; peritracheal hematoma, usually resulting from intraoperative vascular injury to the carotid artery or the internal jugular vein; infections (3\%-8\%); and direct vagus nerve trauma, followed by hoarseness, dyspnea, and dysphagia.,6,10 On the other hand, delayed complications (related to the device and to simulation of the vagus nerve) include delayed arrhythmias, mainly bradycardia and periodic asystolic episodes; laryngopharyngeal dysfunction; respiratory complications during sleep, such as obstructive sleep apnea; stimulation of the phrenic nerve for closer proximity; tonsillar pain mimicking glossopharyngeal neuralgia; and vocal cord damage during prolonged endotracheal intubation. ${ }^{7}$ Some very rare $(<1 \%)$ side effects are pneumothorax, gastrointestinal symptoms such as chronic diarrhea, muscle spasm of the chest wall or sternocleidomastoid muscles, mood changes, drooling, urinary retention, and other cranial nerve palsies, such as Horner syndrome or facial nerve paralysis., ${ }^{3,5,6}$ Finally, two individual case reports described an aggravation of preexisting seizures on increasing VNS output to relatively high levels. ${ }^{5}$ A summary of the most common complications related to VNS is shown in Table 1.

\section{Nerve Section and Neuroma}

The only case describing a partial section of the left vagus nerve with the concomitant formation of a neuroma was reported by Tran et al. ${ }^{9}$ After minor blunt neck trauma, their patient began to experience progressive hoarseness, and subsequently the seizure control worsened. During surgical exploration of the VNS device, they found left vagus nerve injury with compression of the nerve between the positive electrode and anchor tether and a neuroma between the helices of the negative and positive electrodes. Thus, the blunt neck trauma has been considered responsible for a traction injury to the nerve. To date, no complete nerve section has been described. Given her disability, our patient had previously experienced frequent falls, and therefore the device may have pulled the nerve repeatedly. However, in contrast to the findings of Tran et al., ${ }^{9}$ in our case, the section of vagus nerve was distal and far away from the site of the electrode helices. For this reason, we suspect that the rupture of the nerve is more likely due to a direct surgical trauma occurring during the first operation, rather than due to traction exerted on the nerve by the electrodes. This could explain the site of the section and the formation of an amputation neuroma on the proximal end of the nerve. The alternative hypothesis of progressive nerve traction over time could also be considered. Nevertheless, the features of the neuroma found in the revision surgery seemed to be more consistent with a sharp lesion, rather than a progressive tearing induced by repetitive pulling stress. Moreover, at the time of the revision surgery, the strain relief loop of the electrodes was still present, and no traction on the vagus nerve was found.

\section{Laryngopharyngeal Dysfunction}

Side effects related to voice and breathing are the most common ones related to VNS device implant both in children and in adults. $6,7,11,15$ These include alteration in vocal pitch, quality, loudness or vocal effort (hoarseness), dyspnea, and coughing. They are mainly related to stimulation of recurrent laryngeal nerve during VNS and are usually transitory and intermittent. ${ }^{5,6} \mathrm{~A}$ direct correlation has been found between effects on the pharynx and larynx and

TABLE 1. Summary of the most common complications reported in the literature

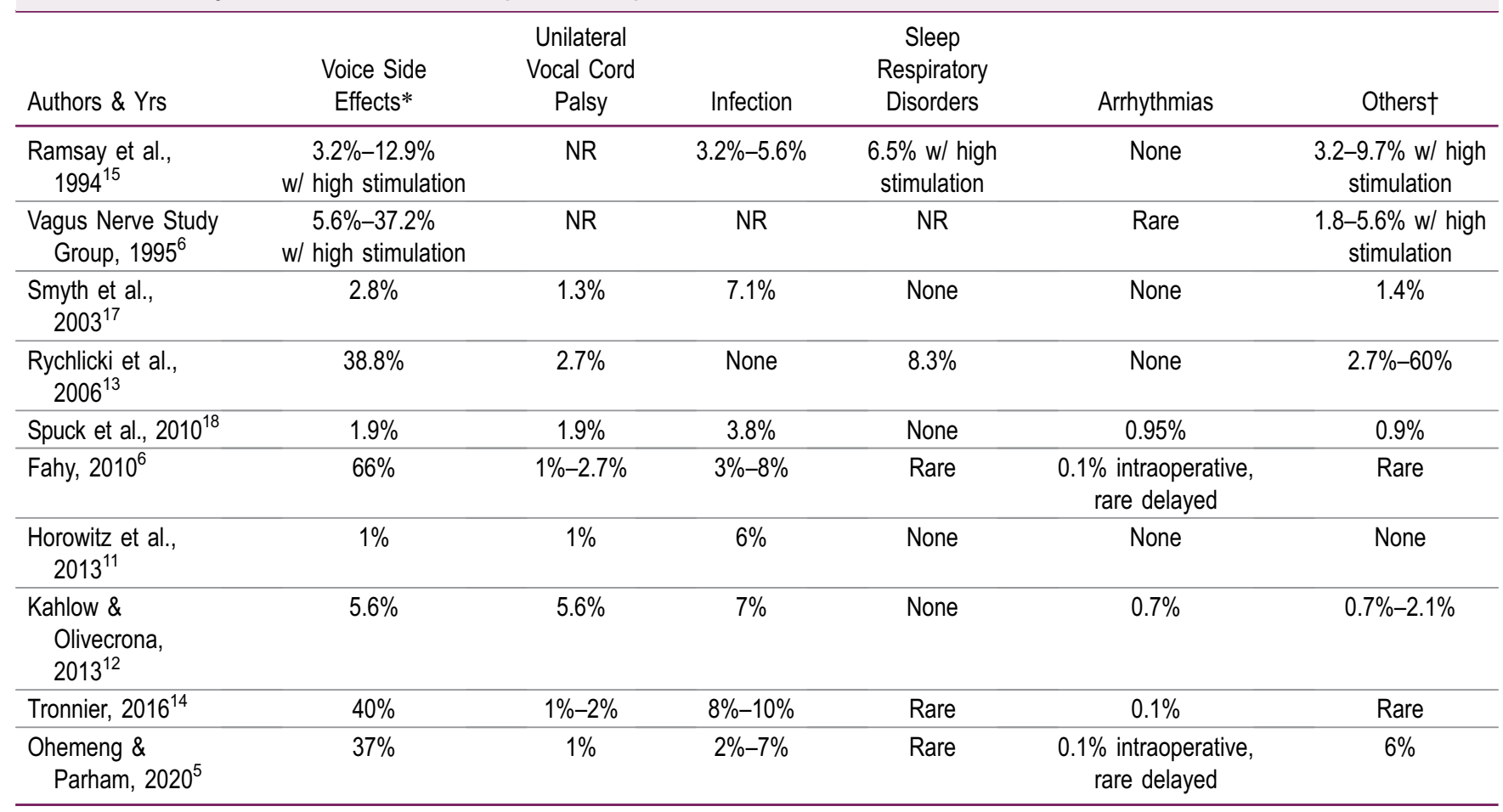

NR $=$ not reported.

* Voice side effects include voice alterations, dyspnea, coughing, dysphagia, and throat pain.

$\dagger$ Other side effects include muscle pain, paresthesia, headache, nerve/vascular injury, keloid development, urinary retention, and mood disturbances. 
frequency of stimulation. Stimuli between $20 \mathrm{~Hz}$ and $30 \mathrm{~Hz}$ cause vocal cord abduction with a small gap during phonation; stimuli of $40 \mathrm{~Hz}$ result in vocal cord adduction; stimuli of $>40 \mathrm{~Hz}$ produce a tetanic adduction with larynx hemispasm. The incidence of voicerelated adverse events is reported to be as high as $66 \%{ }^{6,7}$ The most common serious neurological side effect is unilateral vocal cord paralysis, whose incidence is reported to be around $1 \%{ }^{6}$ it could be due to surgical trauma with direct damage of fibers of the laryngeal nerve inside the vagus nerve or disruption of blood supply, and the effect is usually transitory. ${ }^{5}$ However, decreased vocal cord mobility can be related to repeated intense VNS, resulting in chronic denervation, and usually presents after battery replacement when a stimulus at the same setting can be more intense. Vassilyadi and Strawsburg ${ }^{16}$ reported a delayed onset of vocal cord palsy after VNS explant for infection. The paralysis could be related to nerve inflammation, direct surgical trauma, or reaction to the implant (events not related to nerve stimulation itself). ${ }^{6}$ The delay in the onset of vocal cord palsy could be explained by the fact that the clinical effects of vagus nerve injury, either via surgical manipulation or inflammation, manifest only after a certain degree of demyelination occurs. ${ }^{16}$ In summary, vagus nerve inflammation, surgical trauma, and repeated VNS can be the causes of dysfunction of the vocal cords or folds. This can also lead to aspiration, an event that occurs in patients with epilepsy, even in the absence of VNS; therefore, it is difficult to understand the actual VNS-related contribution. ${ }^{6}$ In our case, hoarseness began immediately after the first surgery and did not recover with time. Moreover, on direct inspection during laryngoscopy 8 years later, the vocal cord was found to be atrophic, confirming that the damage was longstanding. Based on this evidence, we assume that an injury to vagus and recurrent laryngeal nerves should have happened during surgery when the VNS device was first implanted. The damage could have been directly to the nerves or to their blood supply, as previously mentioned. This hypothesis is also reinforced by the finding of the nerve section and neuroma in a location that is distal to the site of the electrodes. In fact, the helices are placed between the site of origin of the superior laryngeal and the recurrent laryngeal nerve. ${ }^{5}$ Cases of vagus nerve damage reported in the literature are summarized in Table 2.

\section{Vocal Cord Paralysis}

Major studies reporting transient left vocal cord paralysis after surgery are those of Smyth et al. in $2003,{ }^{17}$ Rychlicki et al. in $2006,{ }^{13}$ Spuck et al. in 2010, ${ }^{18}$ Horowitz et al. in 2013, ${ }^{11}$ (one case in each series); Ortler et al. in $2010^{19}$ (two cases); and Kahlow et al. in $2013^{12}$ (eight cases). On the contrary, permanent left vocal cord paralysis in VNS is anecdotal. Zalvan et al. ${ }^{20}$ reported one case of vocal cord palsy as result of surgical trauma that did not recover. The possible etiologies of the damage are reduction of blood supply, heating, traction, manipulation, and clamping of the nerve. ${ }^{20}$ Two cases of permanent vocal cord paralysis presented as a consequence of hardware malfunction (outdated systems). Ramsay et al. ${ }^{15}$ illustrated a case of vagus nerve damage due to IPG dysfunction with subsequent abnormal current delivery (higher-voltage, continuous direct current), and Landy et al. ${ }^{21}$ reported one case due to vagus nerve edema determined by lead failure. Another possible cause of irreversible deficit is tampering of the prosthesis by the patients, an event that is more common in those with obesity and patients with cognitive deficits (the so-called "twiddler's syndrome"). For example, Kalkanis et al. ${ }^{22}$ described two cases due to self-inflicted hardware manipulation in
TABLE 2. Cases of nerve damage reported in literature

\begin{tabular}{|c|c|c|}
\hline Authors \& Yrs & $\begin{array}{l}\text { No. of } \\
\text { Case(s) }\end{array}$ & Cause \\
\hline $\begin{array}{l}\text { Landy et al., } \\
\qquad 1993^{21}\end{array}$ & 1 & $\begin{array}{c}\text { Vagus nerve edema caused by } \\
\text { outdated leads }\end{array}$ \\
\hline $\begin{array}{l}\text { Ramsay et al., } \\
1994^{15}\end{array}$ & 1 & $\begin{array}{l}\text { Hardware dysfunction and abnormal } \\
\text { current delivery (outdated IPG) }\end{array}$ \\
\hline $\begin{array}{l}\text { Kalkanis et al., } \\
\qquad 2002^{22}\end{array}$ & 2 & $\begin{array}{l}\text { Rotation of the pulse generator at the } \\
\text { subclavicular pocket by patients } \\
\text { tampering w/ their device }\end{array}$ \\
\hline $\begin{array}{l}\text { Vassilyadi \& } \\
\text { Strawsburg, } \\
2002^{16}\end{array}$ & 1 & $\begin{array}{l}\text { Nerve damage due to wound } \\
\text { infection that spread to deep tissues }\end{array}$ \\
\hline $\begin{array}{l}\text { Zalvan et al., } \\
\qquad 2003^{20}\end{array}$ & 4 & $\begin{array}{l}\text { Damage to the nerve \& its blood } \\
\text { supply during surgery }\end{array}$ \\
\hline $\begin{array}{l}\text { Rijkers et al., } \\
2008^{23}\end{array}$ & 1 & $\begin{array}{l}\text { Blunt neck trauma while performing } \\
\text { martial arts }\end{array}$ \\
\hline $\begin{array}{l}\text { Tran et al., } \\
2011^{9}\end{array}$ & 1 & $\begin{array}{l}\text { Blunt neck trauma \& previous } \\
\text { frequent falls }\end{array}$ \\
\hline $\begin{array}{l}\text { Clark et al., } \\
2012^{10}\end{array}$ & 1 & Leads traction on the vagus nerve \\
\hline Present case & 1 & $\begin{array}{l}\text { Nerve damage due to surgical } \\
\text { manipulation }\end{array}$ \\
\hline
\end{tabular}

patients with mental impairment. Finally, another cause can be direct trauma to the vagus nerve and/or to device; Rijkers et al. ${ }^{23}$ reported one case of permanent paralysis related to VNS dysfunction after neck blunt trauma in martial arts. It should be noted that the incidence of vocal cord palsy is higher in revision surgery than in first implantation $(4.9 \%$ vs. $3.8 \%)^{7}$ As described above, we believe that the permanent vocal cord paralysis of the present case is due to surgical trauma, similar to that described by Zalvan et al. ${ }^{20}$

\section{Lessons}

Medically intractable epilepsy is a debilitating disease, and VNS can provide benefits in terms of seizures control when resections are not feasible or inefficacious. ${ }^{1,2,7}$ After surgical manipulation and chronic stimulation, the left vagus nerve may become more vulnerable, and even minor trauma could lead to irreversible damage, such as complete nerve section. Our case report suggests that the efficacy of VNS stimulation might not be impacted by this kind of lesion. Moreover, we suggest that laryngeal examination should be routinely performed before each VNS surgery to rule out preexisting left vocal cord dysfunction.

\section{References}

1. Morris GL 3rd, Gloss D, Buchhalter J, et al. Evidence-based guideline update: vagus nerve stimulation for the treatment of epilepsy: report of the Guideline Development Subcommittee of the American Academy of Neurology. Neurology. 2013;81(16):1453-1459.

2. Center for Devices and Radiological Health. Summary of safety and effectiveness data (SSED). Stimulator, autonomic nerve, implanted for epilepsy. Accessed March 16, 2019. https://www.accessdata.fda. gov/cdrh_docs/pdf/p970003s207b.pdf

3. US Food and Drug Administration. Summary of safety and effectiveness data (SSED). VNS Therapy ${ }^{\text {TM }}$ System (Depression). Accessed 
October 2, 2019. https://www.accessdata.fda.gov/cdrh_docs/pdf/ P970003S050b.pdf

4. VNS Therapy System Epilepsy Physician's Manual For Healthcare Professionals, August 2020 OUS Version. https://vnstherapy.com/ healthcare-professionals/resources/physician-manuals

5. Ohemeng KK, Parham K. Vagal nerve stimulation: indications, implantation, and outcomes. Otolaryngol Clin North Am. 2020;53(1): 127-143.

6. Fahy BG. Intraoperative and perioperative complications with a vagus nerve stimulation device. J Clin Anesth. 2010;22(3):213-222.

7. Giordano F, Zicca A, Barba $C$, et al. Vagus nerve stimulation: surgical technique of implantation and revision and related morbidity. Epilepsia. 2017;58(suppl 1):85-90.

8. The Vagus Nerve Stimulation Study Group. A randomized controlled trial of chronic vagus nerve stimulation for treatment of medically intractable seizures. Neurology. 1995;45(2):224-230.

9. Tran Y, Shah AK, Mittal S. Lead breakage and vocal cord paralysis following blunt neck trauma in a patient with vagal nerve stimulator. J Neurol Sci. 2011;304(1-2):132-135.

10. Clark AJ, Kuperman RA, Auguste KI, Sun PP. Intractable episodic bradycardia resulting from progressive lead traction in an epileptic child with a vagus nerve stimulator: a delayed complication. J Neurosurg Pediatr. 2012;9(4):389-393.

11. Horowitz G, Amit M, Fried I, et al. Vagal nerve stimulation for refractory epilepsy: the surgical procedure and complications in 100 implantations by a single medical center. Eur Arch Otorhinolaryngol. 2013;270(1):355-358.

12. Kahlow $\mathrm{H}$, Olivecrona $\mathrm{M}$. Complications of vagal nerve stimulation for drug-resistant epilepsy: a single center longitudinal study of 143 patients. Seizure. 2013;22(10):827-833.

13. Rychlicki $F$, Zamponi N, Cesaroni $E$, et al. Complications of vagal nerve stimulation for epilepsy in children. Neurosurg Rev. 2006; 29(2):103-107.

14. Tronnier VM. Vagus nerve stimulation: surgical technique and complications. Prog Neurol Surg. 2015;29:29-38.

15. Ramsay RE, Uthman BM, Augustinsson LE, et al. Vagus nerve stimulation for treatment of partial seizures: 2. safety, side effects, and tolerability. Epilepsia. 1994;35(3):627-636.

16. Vassilyadi M, Strawsburg RH. Delayed onset of vocal cord paralysis after explantation of a vagus nerve stimulator in a child. Childs Nerv Syst. 2003;19(4):261-263.
17. Smyth MD, Tubbs RS, Bebin EM, et al. Complications of chronic vagus nerve stimulation for epilepsy in children. $J$ Neurosurg. 2003;99(3):500-503.

18. Spuck S, Tronnier V, Orosz I, et al. Operative and technical complications of vagus nerve stimulator implantation. Neurosurgery. 2010;67(2 suppl operative):489-494.

19. Ortler M, Unterhofer C, Dobesberger J, et al. Complete removal of vagus nerve stimulator generator and electrodes. J Neurosurg Pediatr. 2010;5(2):191-194.

20. Zalvan C, Sulica L, Wolf S, et al. Laryngopharyngeal dysfunction from the implant vagal nerve stimulator. Laryngoscope. 2003;113(2):221-225.

21. Landy HJ, Ramsay RE, Slater J, et al. Vagus nerve stimulation for complex partial seizures: surgical technique, safety, and efficacy. J Neurosurg. 1993;78(1):26-31.

22. Kalkanis JG, Krishna P, Espinosa JA, Naritoku DK. Self-inflicted vocal cord paralysis in patients with vagus nerve stimulators. Report of two cases. J Neurosurg. 2002;96(5):949-951.

23. Rijkers K, Berfelo MW, Cornips EMJ, Majoie HJ. Hardware failure in vagus nerve stimulation therapy. Acta Neurochir (Wien). 2008;150(4):403-405.

\section{Disclosures}

Dr. Roncon is an employee of PLC, manufacturer of vagus nerve stimulators. Dr. Giordano receives compensation for consulting and he received speaker honorarium from Livanova.

\section{Author Contributions}

Conception and design: Giordano, Noris, Peraio, Zicca. Acquisition of data: Giordano, Noris, Peraio, Zicca. Analysis and interpretation of data: Giordano, Noris, Peraio. Drafting the article: Giordano, Noris, Roncon, Zicca. Critically revising the article: Giordano, Noris, Roncon, Peraio, Zicca, Lenge, Di Rita. Reviewed submitted version of manuscript: Giordano, Noris, Di Rita. Approved the final version of the manuscript on behalf of all authors: Giordano. Administrative/technical/ material support: Giordano, Noris, Lenge. Study supervision: Giordano, Genitori. Operating surgeon: Giordano.

\section{Correspondence}

Flavio Giordano: Meyer Children's Hospital, Florence, Italy. flavio. giordano@meyer.it. 